Correction

\title{
Correction: Gaspari et al. A Simplified Algorithm to Predict Indoor Microclimate in Case of Courtyard Covering. A Case Study for the Courtyard of Palazzo Poggi in Bologna. Eng 2020, 1, 222-239
}

\author{
Jacopo Gaspari * ${ }^{\mathbb{C}}$, Kristian Fabbri $\mathbb{B}^{\mathbb{D}}$, Vincenzo Vodola, Giorgia Ferretti and Lorenzo Indio
}

Citation: Gaspari, J.; Fabbri, K.; Vodola, V.; Ferretti, G.; Indio, L. Correction: Gaspari et al. A

Simplified Algorithm to Predict Indoor Microclimate in Case of Courtyard Covering. A Case Study for the Courtyard of Palazzo Poggi in Bologna. Eng 2020, 1, 222-239. Eng 2021, 2, 227-228. https://doi.org/ $10.3390 /$ eng2020014

Received: 17 May 2021

Accepted: 19 May 2021

Published: 25 May 2021

Publisher's Note: MDPI stays neutral with regard to jurisdictional claims in published maps and institutional affiliations.

Copyright: (c) 2021 by the authors. Licensee MDPI, Basel, Switzerland. This article is an open access article distributed under the terms and conditions of the Creative Commons Attribution (CC BY) license (https:// creativecommons.org/licenses/by/ $4.0 /)$.

Department of Architecture, University of Bologna, 40136 Bologna, Italy; kristian.fabbri@unibo.it (K.F.); vincenzo.vodola2@unibo.it (V.V.); giorgia.ferretti@studio.unibo.it (G.F.); lorenzo.indio@studio.unibo.it (L.I.)

* Correspondence: jacopo.gaspari@unibo.it; Tel.: +39-0547-338349

The authors wish to make the following corrections to this paper [1]:

There was a mistake in Figure 3: as published, the wrong shadow was represented. The corrected Figure 3 appears below.

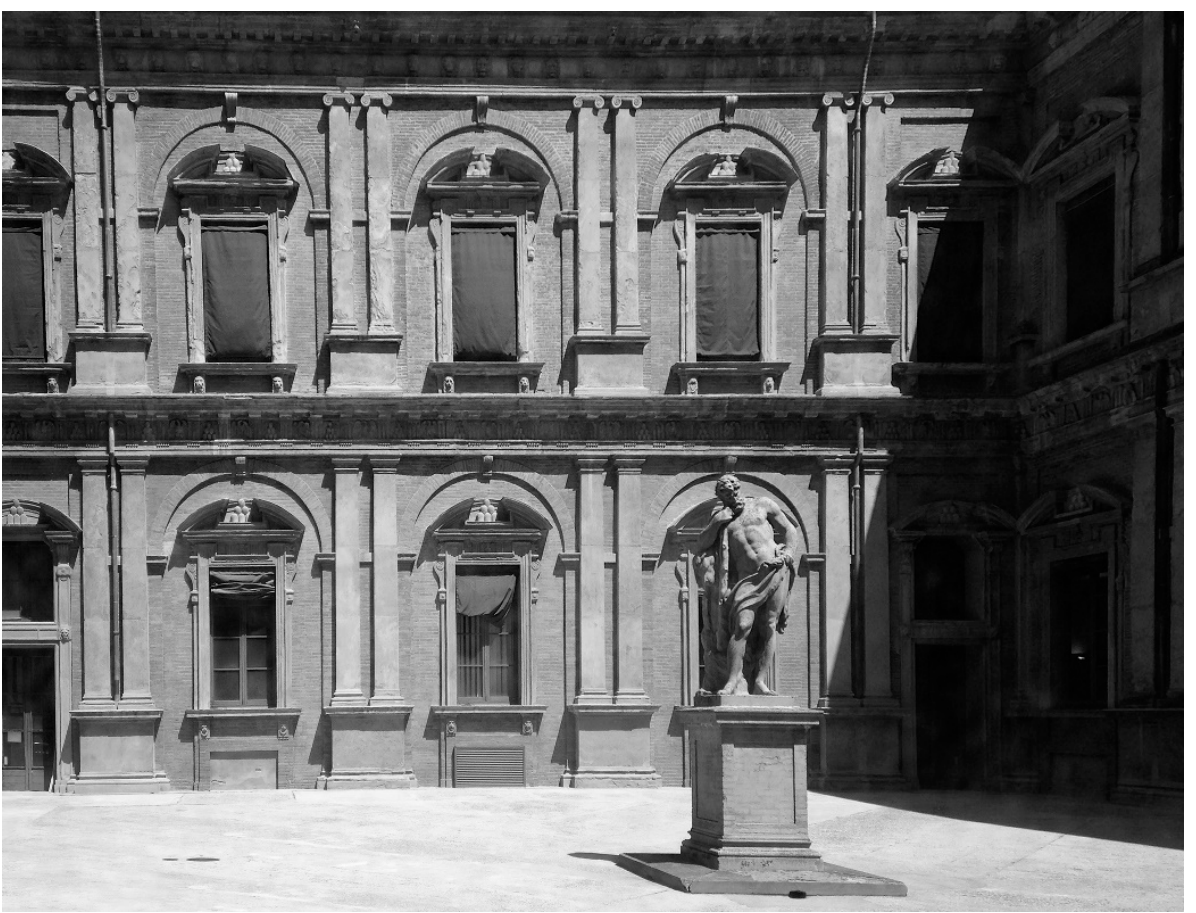

Figure 3. Internal view of Hercules' courtyard (Ferretti, Indio).

There was a mistake in Table A1: as published, the included figures were too small and the source was not listed. In the corrected Table A1, the figures are no longer included. The corrected Table A1 appears below. 
Table A1. Reference cases of covered courtyards after the renovation.

\begin{tabular}{|c|c|c|c|c|}
\hline Reference Case & Location & Designer & Use & Typology \\
\hline $\begin{array}{l}\text { Santa Marta's } \\
\text { military building }\end{array}$ & Verona, Italy & $\begin{array}{l}\text { Massimo Carmassi } \\
\text { Architects }\end{array}$ & Vertical circulation & $\begin{array}{c}\text { glass layer and metal } \\
\text { structure }\end{array}$ \\
\hline British Museum & $\begin{array}{l}\text { London, United } \\
\text { Kingdom }\end{array}$ & Foster and Partners & $\begin{array}{l}\text { Circulation, } \\
\text { additional functions }\end{array}$ & $\begin{array}{l}\text { glass layer and metal } \\
\text { structure }\end{array}$ \\
\hline Maritime Museum & $\begin{array}{l}\text { Amsterdam, The } \\
\text { Netherlands }\end{array}$ & $\begin{array}{l}\text { Ney and Partners, } \\
\text { Dok Arch. Rappange } \\
\text { and Partners }\end{array}$ & New hall & $\begin{array}{c}\text { glass layer and metal } \\
\text { structure }\end{array}$ \\
\hline $\begin{array}{l}\text { Glasdach Staatliche } \\
\text { Bibliothek }\end{array}$ & Passau, Germany & $\begin{array}{l}\text { Awwscz-Westner } \\
\text { Schührer Zöhrer }\end{array}$ & New library hall & $\begin{array}{c}\text { glass layer and metal } \\
\text { structure }\end{array}$ \\
\hline $\begin{array}{l}\text { Museum De } \\
\text { Lakenhal }\end{array}$ & $\begin{array}{l}\text { Leiden, The } \\
\text { Netherlands }\end{array}$ & $\begin{array}{l}\text { Happel Verhoeven, } \\
\text { Julian Harrap } \\
\text { Architects }\end{array}$ & Circulation & $\begin{array}{c}\text { glass layer and metal } \\
\text { structure }\end{array}$ \\
\hline Neus Museum & Berlin, Germany & $\begin{array}{l}\text { David Chipperfield } \\
\text { Architects }\end{array}$ & $\begin{array}{l}\text { Additional space, } \\
\text { exhibition space }\end{array}$ & $\begin{array}{l}\text { glass layer and metal } \\
\text { structure }\end{array}$ \\
\hline Jewish Museum & Berlin, Germany & Daniel Libeskind & $\begin{array}{c}\text { Additional space, } \\
\text { Hall }\end{array}$ & $\begin{array}{l}\text { glass layer and metal } \\
\text { structure }\end{array}$ \\
\hline $\begin{array}{c}\text { Museum of } \\
\text { Hamburg History }\end{array}$ & Hamburg, Germany & $\begin{array}{l}\text { Von Gerkan, Marg } \\
\text { and Partners (gmp) }\end{array}$ & $\begin{array}{c}\text { Additional space, } \\
\text { Hall }\end{array}$ & $\begin{array}{l}\text { glass layer and metal } \\
\text { structure and cables }\end{array}$ \\
\hline Maximilian Museum & Augsburg, Germany & $\begin{array}{l}\text { Hochbauamt der } \\
\text { Stadt Augsburg, } \\
\text { Ludwig and Weiler } \\
\text { Ingenieure }\end{array}$ & $\begin{array}{c}\text { Additional space, } \\
\text { Hall }\end{array}$ & $\begin{array}{c}\text { glass layer } \\
\text { suspended by steel } \\
\text { cables }\end{array}$ \\
\hline Beyazit State Library & Istanbul, Turkey & $\begin{array}{l}\text { Tabanlioglu } \\
\text { Architects }\end{array}$ & $\begin{array}{c}\text { Additional space, } \\
\text { Hall }\end{array}$ & $\begin{array}{l}\text { transparent inflatable } \\
\text { membrane structure }\end{array}$ \\
\hline
\end{tabular}

The authors apologize for any inconvenience caused and state that the scientific conclusions are unaffected. The original article has been updated.

\section{Reference}

1. Gaspari, J.; Fabbri, K.; Vodola, V.; Ferretti, G.; Indio, L. A Simplified Algorithm to Predict Indoor Microclimate in Case of Courtyard Covering. A Case Study for the Courtyard of Palazzo Poggi in Bologna. Eng 2020, 1, 222-239. [CrossRef] 